Printed Version: (ISSN 2090-5262)

Online Version: (ISSN 2090-5270)

March 2013, Volume 3, No. 1 Pages (32 - 40)

\title{
Three Dimensional Biomechanical Analyses of Non-Contact Anterior Cruciate Ligament Injury Mechanisms in Female Volleyball Using Motion Matching Technique
}

\author{
Eman Abdallah Zaid*
}

* Ass. Professor, the department of games, the faculty of physical education for women, Zagazig University,

\begin{abstract}
Volley ball is one of the non-contact sports that is not characterized with injuries to a high extent, specially for ACL injury. Although it has been proved that volleyball female players are exposed to injuries to a medium extent due to sudden movements and shock absorbing in jumps. One of the common injuries in this field is anterior cruciate ligament injuries which have been proven one of the most dangerous injuries in all sports. Methods for analyzing the mechanisms of injuries in sports from video sequences of injury situations are so far limited to a simple visual inspection, which has shown poor accuracy. This is why this study was performed to investigate whether a new model-based image-matching technique could successfully be applied to estimate kinematical characteristics of three typical anterior cruciate ligament (ACL) injury situations in female volleyball. The researcher contacted the sports medicine committee in the volleyball international federation for obtaining injury video incidences from international championships for some world ranked female national teams for the purpose of biomechanical analysis. Videos have been imported to the program Posers 4, where a skeleton model and a model of the surroundings were matched to the background image frame by frame. When the match was considered satisfactory, joint angles as well as velocity and acceleration of the center of mass were calculated. The researcher concluded that the model-based image matching technique can be used to extract kinematical characteristics from videotapes of actual ACL injuries, and may provide valuable information on the mechanisms for ACL injuries in sports. Finally the researcher recommends conducting further studies on other injury incidences for the aim of prevention.
\end{abstract}

Keywords: volleyball - ACL - mechanisms - biomechanics - model.

\section{Introduction and research problem:}

$\mathrm{T}$ he Federation Internationale de Volleyball (FIVB) reports that over 800 million people worldwide play volleyball. Volleyball players must combine vertical and horizontal motion. These athletes must utilize lateral, backward, forward and rotational motion complemented with jumps. The physical properties of the playing surface can further accentuate these demands. Given the growth of beach volleyball as well as court (indoor) volleyball, there are injuries that are distinct to each and common to both. Prevention of injury is best accomplished through preseason strength training and attention to technique during the season. Also many scientists has confirmed the importance of Biomechanics science ( field) in researching and observing the body's motion in order to overcome the mechanical problem and to have critical motional solution for the benefit of the perfect performance. Sports Biomechanics has been identified as the science main tool to understand the nature of human motion through interpreting it to mathematical values. (1: 1) (15) (19)

Volleyball is a sport that requires a lot of jumping and landing which can be very jarring to the body over time. Not surprisingly, most of 
the injuries in the sport come from hitting and blocking. Although more than 380,000 girls play high-school volleyball nationwide, research on female volleyball injuries is as rare as an underhand serve. Fortunately, severe knee ligament injuries are rare in volleyball. (16) Sixty-three percent of injuries are related to jumping. The playing surface clearly impacts injury rates as well as common types of defense and offense maneuvers. (2) (4:5-6) (21)

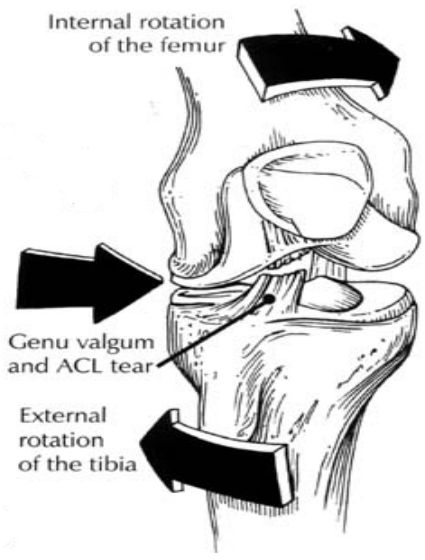

Studies have proved that non-contact anterior cruciate ligament (ACL) injuries can be prevented through training programs focusing on knee control, balance, technique and strength. However, the mechanisms of injury are poorly understood, and controversy exists on the loading patterns involved, which limits our ability to develop improved and targeted prevention programs. $(13: 1)$

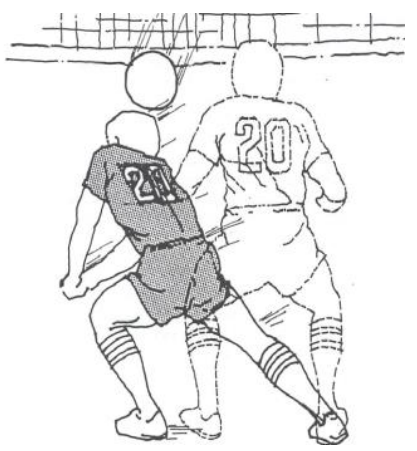

Figure (1)

Partial or complete damage to the anterior cruciate or the medial collateral ligaments due to frequent non contact external rotation of the tibia in landings or defense maneuvers.

Also there are many other reasons for knee injuries in volleyball as in knee bends that are performed repeatedly, and hard landings which causes excess stress on the joints which in turn causes pain and swelling. A common symptom is morning stiffness that decreases as the day progresses. (23)

Data and insight into injury mechanisms can be obtained through different approaches, including surveys of injured athletes, laboratory motion analysis or mathematical simulations. However, with the exception of rare accidents during biomechanical research experiments, the only approach that has the potential to record the kinematics of a real injury situation is analysis of injury videos. In addition to the direct information gained, e.g., knee flexion angles, video analysis is essential to verify whether simulations, whether they are mathematical simulations, cadaver simulations or in vivo simulations, actually correspond to what is seen in real injury situations. Despite the potential significance of obtaining precise motion estimates from actual ACL injury situations, research is so far limited, possibly owing to a lack of precise methods for video analysis.

\section{Material and methods:}

Three ACL injury situations from International volleyball female championships, recorded with un-calibrated cameras, were chosen for analysis. The videos were transformed from their original format into uncompressed AVI sequences before further processing to avoid loss of quality. Then, the sequences were de-interlaced using the Adobe Photoshop (version 4.0). To reconstruct the three-dimensional kinematics of the injured athletes, we utilized a new photogrammetric model-based image-matching technique. The matching was performed using the commercially available program Posers 4 and the Posers Pro Pack. First, models of the surroundings were manually matched to the background for each frame in every camera view, using a key frame and an interpolation technique, by adjusting the camera calibration parameters (position, orientation and focal length). The surroundings were modeled using points, straight lines and curved lines (e.g. volleyball court). We utilized a skeleton model 
from Zygote Media Group Inc. (Provo, Utah, USA) for the athlete matching. This model consisted of 21 rigid segments with a hierarchical structure, using the pelvis as the parent segment. The pelvis motion was described by three rotational and three translational degrees of freedom. The motion of the remaining segments was then described with three rotational degrees of freedom relative to their parent, e.g., the shank relative to the thigh. Normalized ground reaction forces were calculated based on estimated accelerations of the center of mass. The knee and hip joint angles were converted into the joint coordinate system convention of Grood and Suntay (1983), allowed for 57 degrees of freedom. (10)

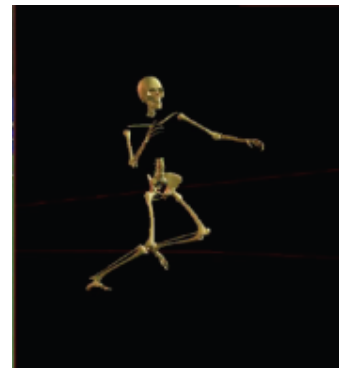

Figure (2)

The skeleton matching model for poser frames with the required matching body segments

The injury incidence videos showed a situation where a female volleyball player developed an ACL injury to the right knee in a one-leg landing. The movies used for the analysis were supplied by the FIVB. The quality was generally very good, although fast-moving body parts were somewhat blurry. The sequence that was matched lasted for approximately $0.8 \mathrm{~s}$. Although other players were visible, they did not occlude the view of the injured player to any significant extent. No anthropometrical measurements were available. The segment dimensions were therefore iteratively adjusted during the matching process until finally, a fixed set of scaling parameters was determined. The center of mass for the segments was then determined by the method of De Leva (1996). Because the shank axial rotation was difficult to assess precisely, we distributed the rotation evenly between the ankle and knee joint, using foot orientation as guidance. (6)

The second injury video showed an ACL injury to the right knee in a female player during a one-leg defense approach movement from right to left. Nevertheless, fast-moving body parts were somewhat blurry, and it was therefore difficult to assess thigh rotation precisely. Other players partly occluded the injured player in one view, but this mainly occurred after the injury presumably had occurred. The skeleton model segment dimensions were set based on these measurements. Again, the axial rotation was evenly distributed between the ankle and knee joint.

The third injury video showed a female player developing an ACL injury to the left knee. The quality was relatively poor because of low contrast. It was difficult to assess thigh rotation. Only a few surrounding landmarks were visible. 


\section{Results:}

\section{First injury:}

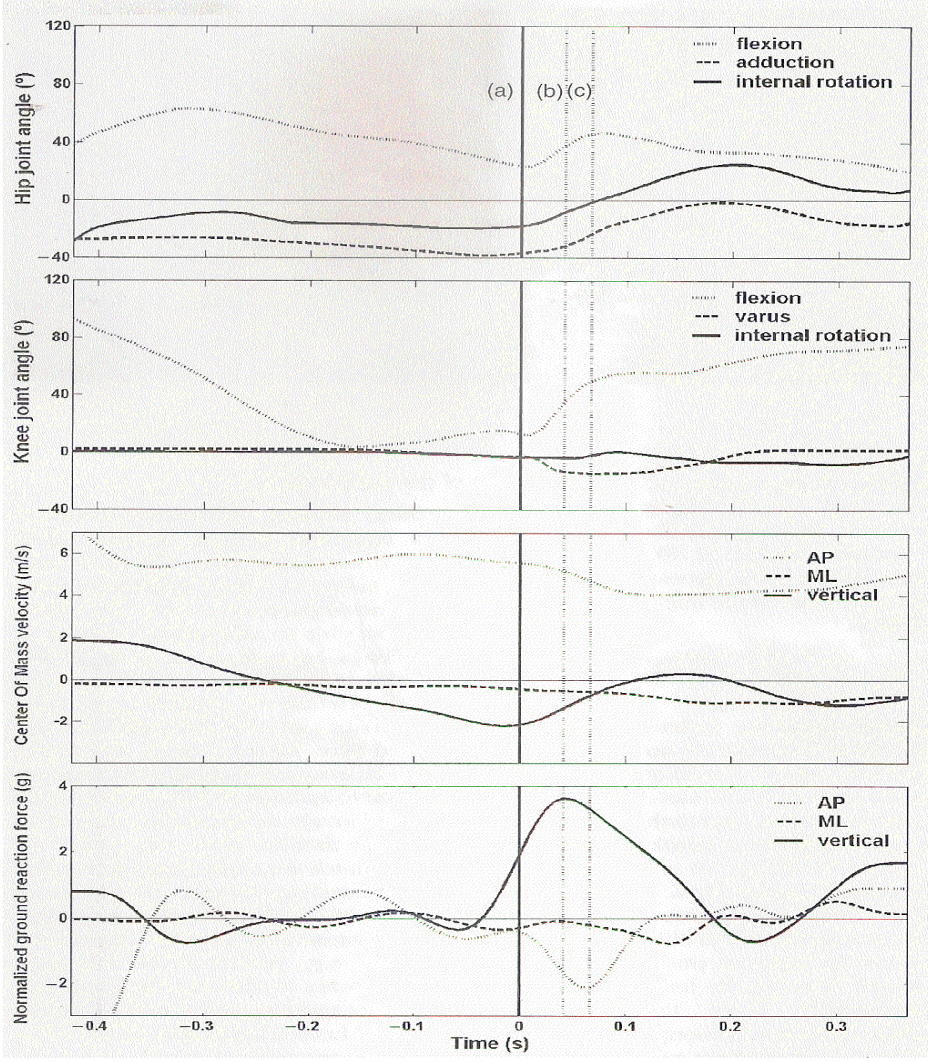

Figure (3)

Time sequence of hip joint angle, knee joint angle, center of mass velocity $(\mathrm{m} / \mathrm{s})$ and normalized ground reaction force $(\mathrm{g})$ of the injured right leg for the first injury situation. The solid vertical line (a) indicates initial ground contact. The two dotted vertical lines indicate the time points $30 \mathrm{~ms}$ (b) and $60 \mathrm{~ms}$ (c) after initial contact, respectively, corresponding to the peak vertical and horizontal force.

The injury occurred when the female player landed on her right leg after hitting the ball in the air. The period before the initial ground contact was a flight phase, although the forefoot touched the ground slightly during the first few frames of the matching. We were able to match successfully both the volleyball court model and the skeleton model to the background image sequence. The visual match was excellent in all frames, i.e. all joints, as well as the feet, hands and head were relatively stable relative to the athlete boundaries. However, the upper extremities appeared slightly less accurate. A good match was also seen in between the positioning of the Poser frames that were seen in the images. The horizontal approach speed was estimated to be $5.6 \mathrm{~m} / \mathrm{s}$, and the cutting angle was 101 . The normalized ground reaction force estimates remained close to zero during the airborne period for the vertical and mediolateral directions, but the antero-posterior force estimate fluctuated somewhat more. At initial contact, the hip flexion angle was estimated to be approximately 231 and knee flexion angle 131. After approximately $30 \mathrm{~ms}$, the vertical force reached its peak at close to four times the body weight. At this point, the hip and knee flexion angles had increased to 381 and 351, respectively, and the valgus angle had increased rapidly to 141 . The knee rotation was less than 51. The maximal antero-posterior force was seen somewhat later: at approximately $60 \mathrm{~ms}$., the body position of the athlete at initial ground contact, and at 30 and $60 \mathrm{~ms}$ after initial contact. 


\section{Second injury}

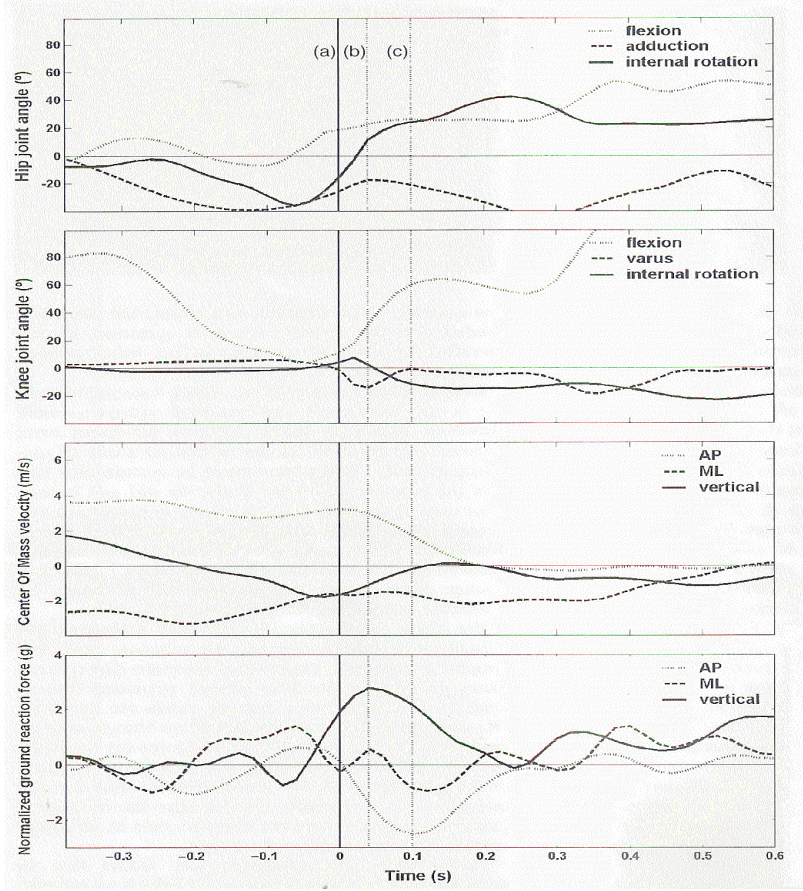

Figure (4)

Time sequence of hip joint angle, knee joint angle, center of mass velocity $(\mathrm{m} / \mathrm{s})$ and normalized ground reaction force $(\mathrm{g})$ of the injured right leg for the second injury situation. The solid vertical line (a) indicates initial ground contact. The two dotted vertical lines indicate the time points $40 \mathrm{~ms}$

(b) and $100 \mathrm{~ms}$ (c) after initial contact, respectively, corresponding to the peak vertical and horizontal force.

The injury occurred when the player performed a side-step cutting maneuver from right to left. We were able to match successfully the court model and the skeleton model to the background image sequence. The visual match appeared excellent in all frames. As for the previous case, all joints, as well as the feet, hands and head were relatively stable relative to the athlete boundaries. However, the match was slightly better for the lower than the upper extremities. The horizontal speed at initial ground contact was estimated to be $3.6 \mathrm{~m} / \mathrm{s}$, and the cutting angle was approximately 671. At initial ground contact, the hip was flexed 191, abducted 261 and externally rotated 161 . The knee was flexed 111 , while rotation and varus-valgus position was neutral. When the vertical force reached its peak at 2.8 times the body weight $40 \mathrm{~ms}$ after initial contact, the hip joint flexion angle was nearly constant, whereas the knee flexion had increased to 311 . At the same time, a peak valgus angle of 151 was seen. The maximal antero-posterior force was seen somewhat later: at $100 \mathrm{~ms}$. The body position of the female player at initial ground contact, and at 40 and $100 \mathrm{~ms}$ after initial contact. During the period before initial ground contact, the player was not completely airborne, as there was slight contact with the contra-lateral foot initially. However, loading during this phase seemed to be minimal, and this assumption is supported by the force estimates. 


\section{Third injury}

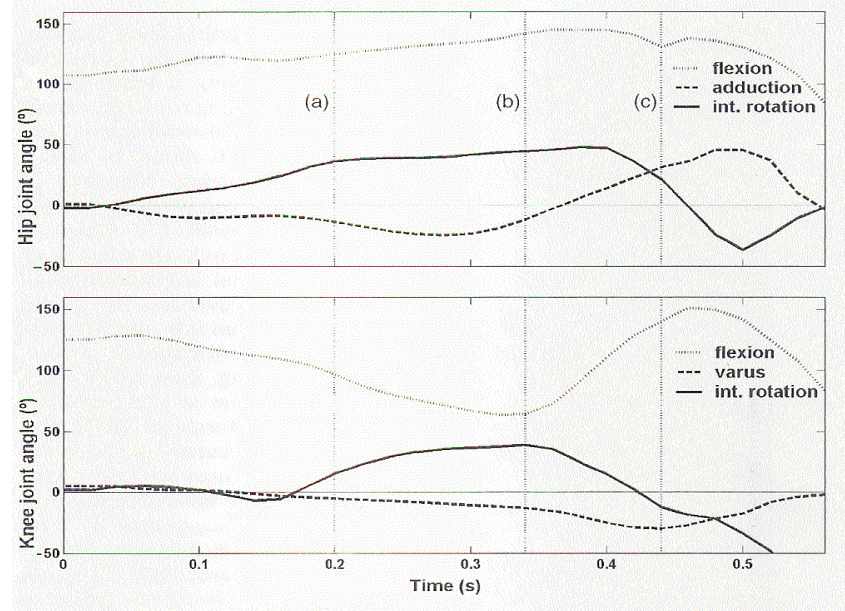

Figure (5)

Hip and knee joint angle history (1) for the injured left leg for the third injury situation. The three dotted vertical lines (a), (b) and (c) indicate the time points 200, 340 and 440 ms into the matched frame sequence, respectively.

We matched $550 \mathrm{~ms}$ of the video sequence, shows the body position of the player 200, 340 and 440 $\mathrm{ms}$ into the sequence. The female player lost her balance during a defense dive, causing her to go into a wide sprawling position. The left knee was then severely twisted, resulting in an ACL tear. The visual match appeared good, but due to the relatively poor video quality, it was somewhat difficult to assess whether the matching was performed correctly at all frames. However, video sequences generated from different viewpoints using different frame rates (i.e., video speed) indicated that the match was satisfactory. While the female player was diving to defend a smash, left hip flexion and internal rotation increased gradually, peaking around $400 \mathrm{~ms}$ at about 1401 and 501, respectively. The knee internal rotation peaked at 401 after $340 \mathrm{~ms}$. At this point, the knee flexion angle was 651 and the valgus angle was 151. The valgus angle then increased to 301 after $440 \mathrm{~ms}$.

\section{Discussion}

Although the model-based image-matching technique is subjective, in the sense that it is dependent on the operator's ability to perform the model matching consistently, this method has shown to be much more accurate than the simple visual inspection approach. The advantages over simple visual inspection of injury videos (Boden et al., 2000; Ebstrup \& Bojsen-Moller, 2000; Teitz, 2001; Olsen et al., 2004) are obvious. First, through computer models, the operator is able to visualize in three dimensions the segment poses that appear to be the best match. Second, the joint angles are calculated automatically. Third, the matching technique can provide continuous descriptions of angular and translational displacements and their derivatives. This greatly expands the possibilities for biomechanical assessments, e.g. determining the time of injury or how different motion patterns may contribute to increased knee loading. The method is also versatile, because it can be used in many different situations, regardless of the motion performed, the camera angle, the number of camera views or whether the camera is stationary or in motion. However, if no known landmarks are present in the background picture, it is not possible to estimate the body velocity and acceleration. Nevertheless, joint kinematics can still be calculated. (3) (7) (20) (17)

For obvious ethical reasons, laboratory measurements of injury situations cannot be performed. Comparing motion estimates with lab trials may even have limited value, as injury situations most likely differ from non-injury situations. Therefore, although it is not possible to evaluate the quality of the current matching by comparing with a high standard, we wanted to investigate whether this technique could successfully be applied to estimate kinematic characteristics of the three ACL 
injury situations presented here. In fact, the quality of the matching can still be assessed using the visual goodness of fit for the surroundings and skeleton models to the actual video footage as guidelines, as well as the timing of airborne motion. However, even if the skeleton joints may match the underlying picture and the match appears excellent, there is no guarantee that the axial rotations of the segments are correct. As our ability to determine axial rotation depends on how well we can see, e.g., surface shape and landmarks, it is also necessary to consider the quality of the input. Poor picture quality, partial occlusion, low sampling rate, lack of known landmarks in the video image or having only one camera may lead to suboptimal matching. The more usable information available, the easier it will be to discover errors in the motion patterns. However, more information also means that producing a consistent continuous motion throughout the sequence is made even more challenging. Of the three injury situations studied, the video sequences represent the best input that can be expected from real injury situations, enabling us to position the athlete correctly in space. After several iterations of manual fitting, the model of the court matched very well in all of the views throughout the sequence. In contrast, we could not estimate the center of mass velocity and acceleration in the third injury video because there were no known landmarks in this video sequence.

The matching of the skeleton to the video image of the athlete appeared to be excellent for first two injury situations, achieving a good fit with the skeleton model joints in all frames. Even so, it is likely that axial rotations of the thigh and shank were less accurate than hip and knee flexion and abduction angles, as matching rotational orientation is more difficult because of the shape of the segments, lack of visible landmarks and also skin motion that occurs relative to the underlying bones. The athlete's clothing also limits our ability to assess pelvis and thigh orientation precisely. As shown in many recent laboratory studies, estimating pelvic rotation is also difficult although having multiple video filming both in the floor plane and from above is helpful, as was the case for the first two injury situations. In contrast, determining joint motion that does not occur in the video plane is more difficult (e.g., spine flexion and knee flexion). Although the model-based image technique cannot provide perfect estimates in all situations, sensitivity testing showed that the valgus estimates during the first $30 \mathrm{~ms}$ of the $1^{\text {st }}$ injury matching and $40 \mathrm{~ms}$ of the $2^{\text {nd }}$ matching were relatively robust, as varying femoral rotation did not substantially affect the estimated valgus angle at low knee flexion angles. For the $3^{\text {rd }}$ injury situation, the poorer video quality made it more difficult to interpret the segment poses for individual frames. But after several iterations assessing the skier in different views, a satisfactory result was achieved, which was consistent throughout the matching. One advantage when matching the third injury situation is that shank rotation can probably be matched quite accurately based on court landmarks orientation. However, assessing thigh axial rotation was quite difficult and valgus angle estimates must be interpreted with caution, as they are highly dependent on thigh rotation in this flexed position. Owing to the possible errors in such low-quality, injury situations, the accuracy needed for the specific research question should therefore be carefully assessed before being considered for analysis. Foot orientation guided the shank rotation matching in the $1^{\text {st }}$ and $2^{\text {nd }}$ videos as well. However, although shank rotation estimates were shown to be excellent in the validation study, we cannot be certain that this was the case here as well.

First, we did not have a clear view of the foot attitude in any of the situations. Second, the assumption that the rotation is evenly distributed between the knee and ankle joint has not been validated, and should be tested in laboratory studies. Third, this relationship will probably be altered in case of an ACL injury. We have previously shown that estimates for velocity and acceleration can be achieved with reasonable accuracy in the vertical direction (for video views parallel to the floor plane). Based on this, it may be assumed that the acceleration estimates for the matching is at least as accurate as the laboratory results. Another indication that the estimates are reasonably good is that the calculated ground reaction forces are close to zero in all directions before initial ground contact. 
Thus, the proposed model-based image-matching technique at present provides a suitable framework for utilizing the available information optimally in injury situations, where controlled experimental techniques cannot be used. We chose to analyze ACL injury videos where such injuries are proven frequent, and we aimed to represent the range of video quality that can be expected from injuries occurring during TV broadcasts. Although previous studies reported in the literature using simple visual inspection of injury videotapes hypothesize that non-contact ACL injuries in team/ball sports occur at a flexion angle of less than 301 (Boden et al., 2000; Kirkendall \& Garrett, 2000; Teitz, 2001; Olsen et al., 2004), this methodology has been shown to have poor precision and accuracy. A key limitation of the visual inspection method is that it is not possible to determine the exact timing of the rupture. One potential advantage of the current model-based image-matching technique is that it may be possible to estimate the timing of the injury with greater precision using different criteria, i.e. by determining when the joint configuration becomes abnormal, by observing sudden changes in the joint angular motion and by assessing the ground reaction forces. When examining the time course for knee joint angles in the $1^{\text {st }}$ and $2^{\text {nd }}$ cases, the valgus angle increased abruptly, increasing from 41 to 151 within $30 \mathrm{~ms}$ and from 31 to 161 within 40 ms, respectively. (3) (14) (20) (18)

However, at this point it is not known how the ground reaction forces correspond to ACL loading, although the in case study of Cerulli et al. (2003) indicates some degree of ACL force increase at the vertical ground reaction force peak. Nevertheless, these results indicate that valgus loading on a relatively straight leg (from about 151 to 401) may be a key causative factor. This interpretation supports previous theories from video studies, and also agrees with recent findings by Hewett et al. (2005), who showed that female athletes who tended to land with increased dynamic valgus and high abduction loads are at increased risk of ACL injuries. (5) (11)

However, analysis of a larger sample of cases and comparisons of injury vs non-injury situations are necessary to evaluate whether this is indeed a general trend or merely a result of estimate errors or a non-typical situation.

\section{References:}

1- R. Bahr, 1. A. Bahr, Incidence of acute volleyball injuries, a prospective cohort study of injury mechanisms and risk factors, Scund J Med Sci Sports 1997. 7: 166-171

2- Beverly Oden, Avoid Volleyball's Most Common Injuries, About.com guide, 2012.

3- Boden BP, Dean GS, Feagin JA Jr, Garrett WE Jr. Mechanisms of anterior cruciate ligament injury. Orthopedics 2000: 23: 573-578.

4- Briner WW Jr, Kacmar L., Common injuries in volleyball. Mechanisms of injury, prevention and rehabilitation. Sports Med. 1997 Jul;24(1):65-71.

5- Cerulli G, Benoit DL, Lamontagne M, Caraffa A, Liti A. In vivo anterior cruciate ligament strain behaviour during a rapid deceleration movement: case report. Knee Surg Sports Traumatol Arthrosc 2003: 11: 307-311.

6- De Leva P. Adjustments to Zatsiorsky- Seluyanov's segment inertia parameters. J Biomech 1996: 29: 1223-1230.

7- Ebstrup JF, Bojsen-Moller F. Anterior cruciate ligament injury in indoor ball games.Scand J Med Sci Sports 2000:10: 114-116.

8- Grood ES, Suntay WJ. A joint coordinate system for the clinical description of threedimensional motions: application to the knee. J Biomech Eng 1983: 105:136-144.

9- Hewett TE, Myer GD, Ford KR, Heidt RS Jr, Colosimo AJ, McLean SG, Van Den Bogert AJ, Paterno MV, Succop P. Biomechanical measures of neuromuscular control and valgus loading of the knee predict anterior cruciate ligament injury risk in female athletes. A prospective study. Am J Sports Med 2005: 33: 492-501. 
10- Jonathan C. Reeser, Roald Bahr, Principles of prevention and treatment of common volleyball injuries, FIVB medical committee, 2011.

11- Kirkendall DT, Garrett WE Jr. The anterior cruciate ligament enigma. Injury mechanisms and prevention. Clin Orthop Relat Res 2000: 372: 64-68.

12- Mark A. Caselli, Secrets to treating lower extremity volleyball injuries, Volume 18 - Issue 10 - October 2005

13- Madison, W, Increase in girls' volleyball players ups risk for injury, 2009.

14- Olsen OE, Myklebust G, Engebretsen L, Bahr R. Injury mechanisms for anterior cruciate ligament injuries in team handball: a systematic video analysis. Am J Sports Med 2004: 32:1002-1012.

15- Olsen OE, Myklebust G, Engebretsen L, Holme I, Bahr R. Exercises to prevent lower limb injuries in youth sports: cluster randomised controlled trial. Br Med J 2005: 330: 449.

16-Schafle MD, Common injuries in volleyball. Treatment, prevention and rehabilitation, Sports Med. 1993 Aug;16(2):126-9.

17- Teitz CC. Video analysis of ACL injuries. IN: In: Griffin LY, ed. Prevention of Noncontact ACL injuries.Roesmont, Ill: American Academy of Orthopaedic Surgeons; 2001:93-96..

18- Thomas Souza, Volleyball injuries, Dynamic Chiropractic - May 1, 2000, Vol. 18, Issue 10.

19- www.fivb.org, 2012

20- www.madform.com, Common volleyball injuries, 2011 